Correction to J. Electron. Mater., vol. 34, no. 6, June 2005, pp. 949-952.

The authorship of this paper should read as follows:

\title{
P-Type Doping Utilizing Nitrogen and Mn Doping of ZnO Using MOCVD for Ultraviolet Lasers and Spintronic Applications
}

\author{
E. JAMES EGERTON ${ }^{1}$ ASHOK K. SOOD,${ }^{1,5}$ RAJWINDER SINGH, ${ }^{1}$ \\ YASH R. PURI, ${ }^{1,2}$ DAVID C. LOOK, ${ }^{3}$ AND TODD STEINER ${ }^{4}$ \\ 1.-Magnolia Optical Technoologies, Inc., Woburn, MA 01801. 2.-Also College of Management, \\ University of Massachusetts-Lowell, Lowell, MA -1854. 3.-Semiconductor Research Center, \\ Wright State University, Dayton, $\mathrm{OH}$ 45435. 4.-AFOSR/NE, Arlington, VA 22203-1956. \\ 5._E-mail: aksood@magnoliaoptical.com.
}

$\mathrm{ZnO}$ has distinct advantages over competing technologies such as GaN. Two advantages are the inherent improvement in ultraviolet (UV) brightness, necessary for the biological sensor application where the signal-to-noise ratio (SNR) is enhanced by a bright UV source, and the second is the availability of $\mathrm{ZnO}$ lattice-matched substrates, which will result in lower defect densities than GaN, higher manufacturing yield, and then lower cost. The $\mathrm{ZnO}$ material system's advantage in exciton binding energy of $60 \mathrm{MEV}$, a three-time improvement over GaN, will result in UV emitters with superior performance. ${ }^{1}$

Key words: $\mathrm{ZnO}$, p-type, doping 